\title{
Prelacteal feeding and associated factors in Ethiopia: systematic review and meta- analysis
}

\author{
Habtamu Temesgen ${ }^{1 *}$, Ayenew Negesse ${ }^{1}$, Wubetu Woyraw $^{1}$, Temesgen Getaneh² and Molla Yigizaw ${ }^{3}$
}

\begin{abstract}
Background: Prelacteal feeding can be defined as giving any solid or liquid foods other than breast milk during the first three days after birth. It affects timely initiation of breastfeeding and exclusive breastfeeding practices. Even though the issue was investigated in Ethiopia, fragmented and inconsistent findings were reported. Therefore, the main objective of this meta-analysis was to estimate the pooled prevalence of prelacteal feeding and associated factors in Ethiopia.

Methods: The preferred reporting items for systematic reviews and meta-analyses guideline was followed. Articles were systematically searched through different searching mechanisms. Joanna Briggs Institute Meta-Analysis of Statistics Assessment and Review Instrument adapted for cross-sectional study design was used for quality assessment of each individual study. The total of 28 studies were included and analyzed. The random effect model was used to estimate the pooled prevalence; subgroup analysis and meta-regression were performed to identify the probable source of heterogeneity. Both Egger's, and Begg's test were used to check publication bias. The effects between associated factor variables, and prelacteal feeding practices were tested.
\end{abstract}

Results: A total of 492 studies were retrieved and 28 studies were included in the meta-analysis. The pooled prevalence of prelacteal feeding practice in Ethiopia was $25.29 \%$ (95\% Confidence Interval [CI] 17.43, 33.15) with severe heterogeneity $\left(I^{2}=99.7, p<0.001\right)$ and no publication bias. Antenatal care (Odds Ratio [OR] $\left.0.25,95 \% \mathrm{Cl} 0.09,0.69\right)$, counselling on infant feeding (OR 0.37, 95\% Cl 0.22, 0.63), timely initiation of breastfeeding (OR $0.28,95 \% \mathrm{Cl} 0.21,0.38$ ) and an urban residence (OR 0.47, 95\% Cl 0.26, 0.86) had lower odds, while home birth had higher odds (OR 3.93, 95\% Cl 2.17, 7.10) of prelacteal feeding in Ethiopia.

Conclusions: In Ethiopia, one in four children were given prelacteal foods. Mothers who gave birth at home are more prone to give prelacteal foods. Whereas, antenatal care, timely initiation of breastfeeding, counseling on infant feeding and an urban residence decreases prelacteal feeding practices in Ethiopia. Therefore, the government and health institutions should focus to increase maternal health service utilization and promote infant and young child feeding practices according to the guideline.

Keywords: Prelacteal feeding, Pooled prevalence, Ethiopia, Associated factors

\footnotetext{
* Correspondence: habtamutem@gmail.com

${ }^{1}$ Department of Human Nutrition and Food Sciences, College of Health

Science, Debre Markos University, Debre Markos, Ethiopia

Full list of author information is available at the end of the article
}

(c) The Author(s). 2018 Open Access This article is distributed under the terms of the Creative Commons Attribution 4.0 International License (http://creativecommons.org/licenses/by/4.0/), which permits unrestricted use, distribution, and reproduction in any medium, provided you give appropriate credit to the original author(s) and the source, provide a link to the Creative Commons license, and indicate if changes were made. The Creative Commons Public Domain Dedication waiver (http://creativecommons.org/publicdomain/zero/1.0/) applies to the data made available in this article, unless otherwise stated. 


\section{Background}

Prelacteal feeding is giving any solid or liquid foods other than breast milk during the first 3 days after birth [1-3]. Even though the World Health Organization (WHO) recommends exclusive breastfeeding (EBF) for the first 6 months, 823,000 children under 5 years of age, annually, were suffering from improper breastfeeding practice including prelacteal feeding $[4,5]$ and every day, 3000 up to 4000 infants die in the developing world from diarrhea and acute respiratory infections $[6,7]$.

Prelacteal feeding affects the timely initiation of breastfeeding and exclusive breastfeeding [3]. Globally, suboptimal infant feeding, including prelacteal feeding contributes $45 \%$ of neonatal mortality, $30 \%$ of diarrheal mortality and $18 \%$ of acute respiratory deaths $[8,9]$. Prelacteal feeding reduces the immunological benefits that gains from colostrum and increases the risk of susceptibility to infection [10]. Furthermore, directly it predisposes newborns to pathogenic contaminants creates physiological disruptions in the immature gastrointestinal system and discourages newborns from initiating breastfeeding. In addition, mother-baby bonding may be interrupted and interfering with breast milk production [10-13].

Prelacteal feeding practice is a predominant problem in the developing world. Prelacteal feeding practice in Vietnam and India was 73.3 and $40.1 \%$ respectively [10, 14]. A study in Africa revealed that about $32.2 \%$ in Sub-Saharan [15], 60\% in Egypt [16], 31.3\% in Uganda [17] of mothers practiced prelacteal feeding. Data from the Ethiopian Demographic Health Survey (EDHS) of 2011 report showed that $27 \%$ of infants were given prelacteal feedings within the first 3 days of life [18]. Also, the national survey revealed that prelacteal feeding was 28.9\% in Ethiopia [19].

In Ethiopian, different independent and fragmented studies have been conducted to assess the magnitude of prelacteal feeding practice and its determinants. These discrete studies reported that the magnitude of prelacteal feeding in Ethiopian were ranging from $5.9 \%$ up to $75.8 \%[20,21]$. Prelacteal feeding practice is a well-documented phenomenon and need to be the focus area of research to determine the prevalence and its predictors in Ethiopia. A number of researchers have reported the prevalence of prelacteal feeding practices in Ethiopia [7, 9, 13, 20-43].

Those individual studies indicated that there is great variation and inconsistencies of prelacteal feeding and predictors in Ethiopia. The common factors reported by the above studies were place of residence, place of delivery, antenatal care, counselling on feeding and time to initiate breastfeeding $[7,9,13,20,22-25,27$, $28,30,34,41]$. The reasons for disparity of prelacteal feeding practice and its predictors in Ethiopia have not yet been investigated. In addition to this gap, there are no documented data on pooled prevalence of the prelacteal feeding practice in Ethiopia. Therefore, the main objective of this systematic review and meta-analysis was to estimate the pooled prevalence of prelacteal feeding practice and its associated factors in Ethiopia.

The findings of this study will be an input to policy makers and program planners of the Ethiopian government to design appropriate interventions to decrease prelacteal feeding practice and also important to intervene important predictors to reduce prelacteal feeding practices. This review also will give the national figure for future researchers.

\section{Methods \\ Searching strategies}

This systemic review and meta-analysis were designed to estimate the pooled prevalence of prelacteal feeding and its associated factors of prelacteal feeding in Ethiopia. Initially meta-analysis and systematic reviews, including registered protocols were searched to avoid duplications. It confirmed that there was no review and meta-analysis conducted related to prelacteal feeding in Ethiopia. Published research reports of prelacteal feeding and its associated factors were searched. We systematically reviewed and analyzed published research articles to determine the pooled prevalence of the prelacteal feeding practice and its factors in Ethiopia. To identify published articles, major databases PUBMED/MEDLINE, Cochrane library, Google and Google Scholar were used. In addition, reference lists were used. The key term used in PubMed search was "prevalence" OR "magnitude" AND "prelacteal" AND "feeding practice" AND "Ethiopia" AND "associated factors" AND "age less than five years". The search was conducted from May, 2018 to June 30, 2018.We followed the Preferred Reporting Items for Systematic Reviews and Meta-Analyses (PRISMA) guideline during the systematic review [44].

\section{Inclusion criteria \\ Study scope}

All studies which report the prevalence of prelacteal feeding and associated factors of prelacteal feeding in Ethiopia were included under this systematic review and meta-analysis.

\section{Study design}

Cross-sectional study design was included. 


\section{Language}

Articles published in the English language were included.

\section{Population}

All studies conducted in Ethiopia were considered.

\section{Publication and publication year}

Published articles until June/ 2018 were included.

\section{Exclusion criteria}

Articles published other than in English language and studies which didn't report specific outcomes for prelacteal feeding were excluded.

\section{Data abstraction}

The database search results were collected and duplicate articles were removed manually using endnote (version X7). Data were extracted by two authors using a standardized data extraction spread sheet. Data extraction sheet included study characteristics such as: (1) Authors' name, region, study year, publication year, study design, study setting, sample size, response rate, studies' quality score and sampling; (2) prevalence of prelacteal feeding; (3) residence, antenatal care, place of delivery, initiation of breastfeeding within 1 hours were also extracted from each individual study. Those categorical variables tabulated ( $\mathrm{a}, \mathrm{b}, \mathrm{c}$ and d) with prelacteal feeding during abstraction.

\section{Quality assessment (appraisal) of studies}

The database search results were combined and duplicate articles were removed manually using endnote (version X7). Joanna Briggs Institute Meta-Analysis of Statistics Assessment and Review Instrument (JBI-MAStARI) adapted for both cross-sectional/case-control study design was used [45]. Three independent reviewers critically evaluated each individual paper. Discrepancies between those reviewers were solved by discussion. If not, a third reviewer was involved to resolve inconsistencies in between the two independent reviewers. Studies, which score five and above from a total of nine scores were included in the final systematic review and meta-analysis.

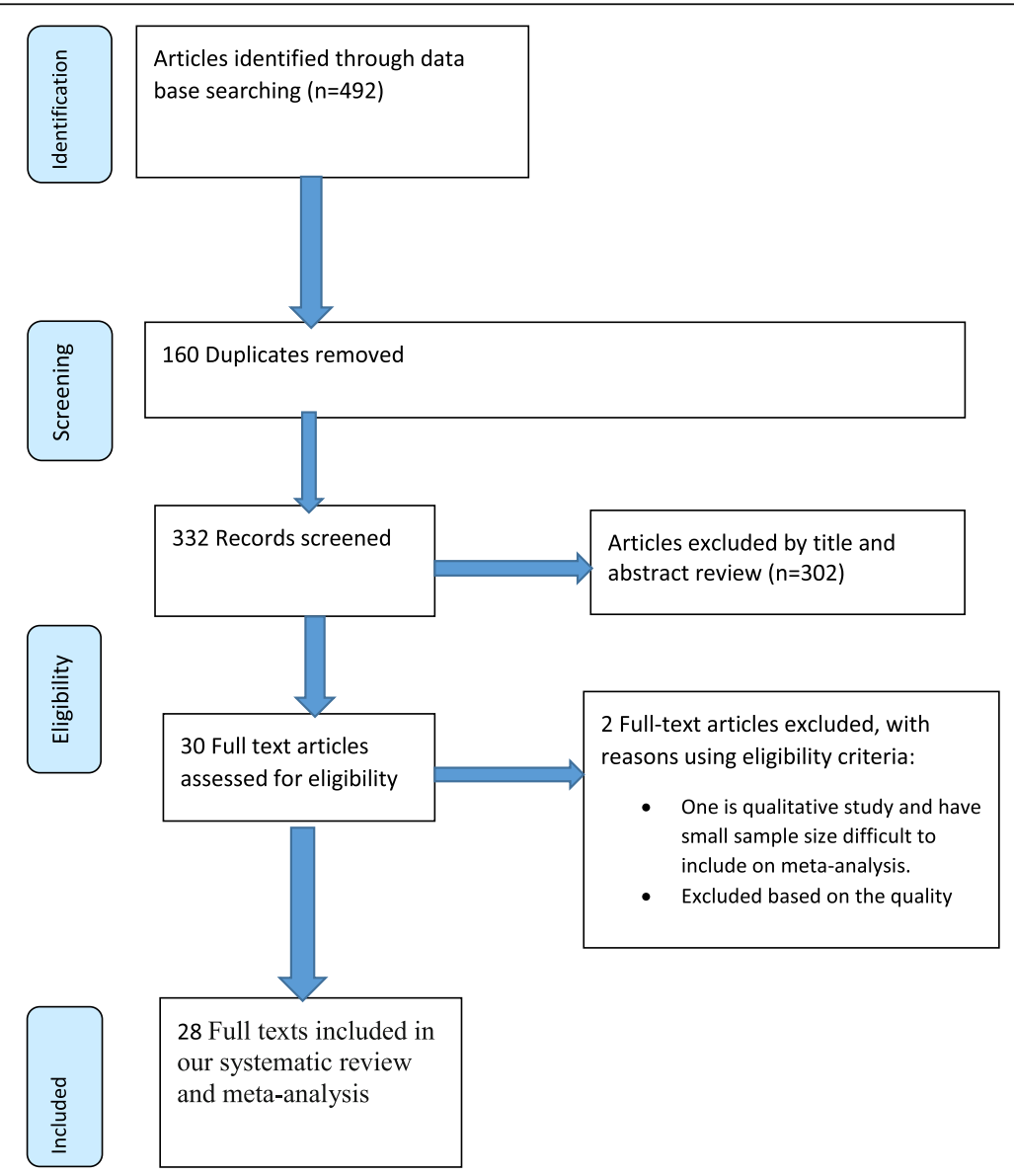

Fig. 1 PRISMA flow diagram of included studies to estimate the pooled prevalence of prelacteal feeding practices and its predictors in Ethiopia 


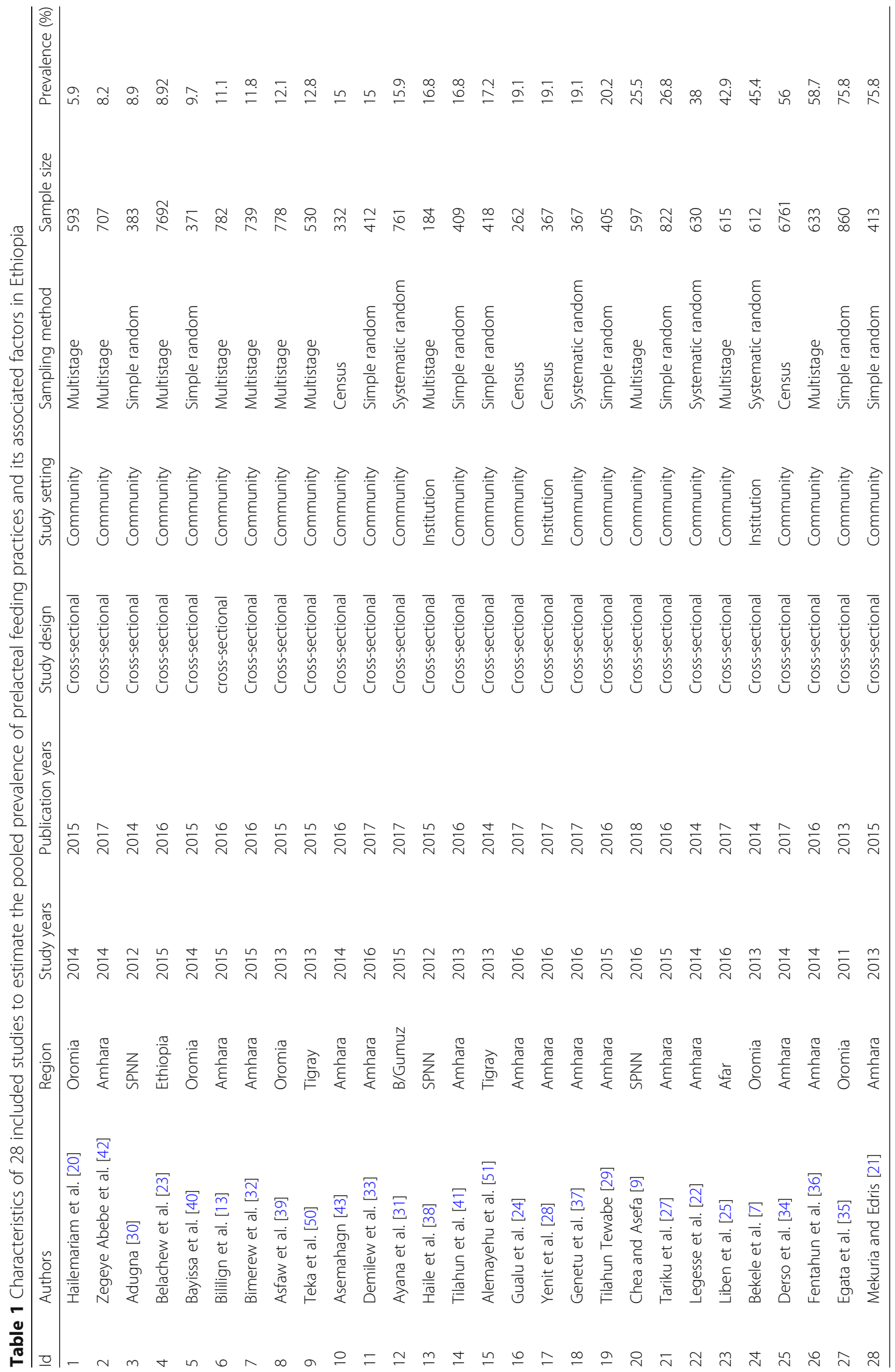




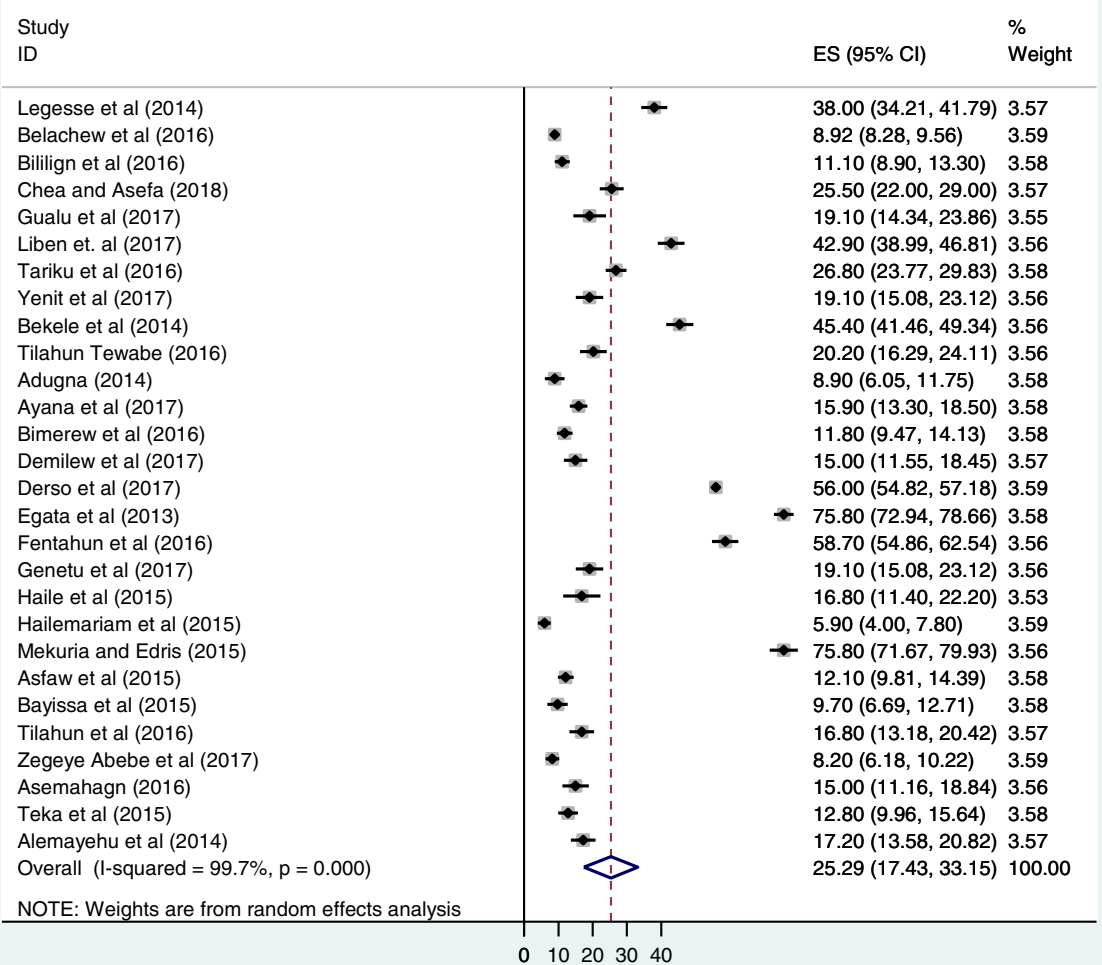

Fig. 2 Forest plots showing the pooled prevalence of prelacteal feeding practice in Ethiopia

Table 2 Subgroup analysis which indicates the pooled prevalence prelacteal feeding practices in Ethiopia

\begin{tabular}{|c|c|c|c|c|c|c|c|}
\hline Subgroups & & $\begin{array}{l}\text { Number of studies } \\
\text { included }\end{array}$ & Prevalence (95\% CI) & $\begin{array}{l}\text { Heterogeneity } \\
\text { statistics }\end{array}$ & $p$-value & $1^{2}$ & Tau-squared \\
\hline \multirow[t]{4}{*}{ Region } & Amhara & 15 & $27.37(15.50,39.25)$ & 3764.65 & $<0.001$ & $99.6 \%$ & 547.17 \\
\hline & Oromia & 5 & $29.76(4.04,55.49)$ & 1904.20 & $<0.001$ & $99.8 \%$ & 859.03 \\
\hline & SNNP & 3 & $17.04(5.97,28.11)$ & 52.18 & $<0.001$ & $96.2 \%$ & 91.43 \\
\hline & ${ }^{\mathrm{a}}$ Others & 5 & $19.43(10.17,28.69)$ & 319.43 & $<0.001$ & $98.7 \%$ & 109.31 \\
\hline \multirow{2}{*}{$\begin{array}{l}\text { Time of study } \\
\text { years }\end{array}$} & Before 2015 & 15 & $30.94(17.51,44.37)$ & 5314.10 & $<0.001$ & $99.7 \%$ & 701.28 \\
\hline & 2015 and above & 13 & $18.70(14.10,23.30)$ & 543.91 & $<0.001$ & $97.8 \%$ & 68.67 \\
\hline \multirow[t]{3}{*}{ Study setting } & Community based & 25 & $25.07(16.67,33.48)$ & 8171.93 & $<0.001$ & $99.7 \%$ & 456.90 \\
\hline & Institution based & 3 & $27.15(8.37,45.92)$ & 109.26 & $<0.001$ & $98.2 \%$ & 269.91 \\
\hline & $\begin{array}{l}\text { Systematic random } \\
\text { sampling }\end{array}$ & 4 & $29.57(15.19,43.94)$ & 200.50 & $<0.001$ & $98.5 \%$ & 211.74 \\
\hline \multirow[t]{3}{*}{$\begin{array}{l}\text { Sampling } \\
\text { techniques }\end{array}$} & $\begin{array}{l}\text { Multistage random } \\
\text { sampling }\end{array}$ & 11 & $19.38(13.10,25.66)$ & 999.06 & $<0.001$ & $99.0 \%$ & 110.54 \\
\hline & $\begin{array}{l}\text { Simple random } \\
\text { sampling }\end{array}$ & 9 & $29.57(11.93,47.22)$ & 2037.82 & $<0.001$ & $99.6 \%$ & 726.33 \\
\hline & Census & 4 & $27.34(1.61,53.08)$ & 798.42 & $<0.001$ & $99.6 \%$ & 686.01 \\
\hline
\end{tabular}

${ }^{\mathrm{a}}$ Tigray, B/gumuz, Afar and national study 
Table 3 Meta regression to identify source of heterogeneity for the prevalence of prelacteal feeding practices in Ethiopia

\begin{tabular}{lllll}
\hline Variables & & No & Coefficients & $p$-value \\
\hline Study year & 2015 or later & 13 & Reference & Reference \\
& Before 2015 & 15 & 12.15842 & 0.111 \\
Sample & Sample size & 28 & 0.0011158 & 0.617 \\
Study setting & Community based & 25 & -2.059248 & 0.871 \\
& Institution based & 3 & Reference & Reference \\
Sampling & Censes & 4 & Reference & Reference \\
techniques & Multistage & 11 & 12.05831 & 0.519 \\
& Simple random & 9 & 12.41248 & 0.861 \\
& Systematic random & 4 & 14.6074 & 0.881 \\
\hline
\end{tabular}

\section{Outcome measurements}

This review and meta-analysis have two main outcomes. The primary outcome was prevalence of prelacteal feeding practices. The second outcome was factors associated with prelacteal feeding practices in Ethiopia.

\section{Data analysis}

The extracted data were entered into an excel sheet and imported to STATA version 14 for analysis. Heterogeneity among reported prevalence was assessed by using the inverse variance $\left(\mathrm{I}^{2}\right)$ with Cochran $\mathrm{Q}$ statistic of 25, 50 and $75 \%$ as low, moderate and sever heterogeneity respectively with $p$ - value less than 0.05 [46]. Random effects meta-analysis model was used to estimate the pooled prevalence of prelacteal feeding. The forest plot was also used to visualize the presence of heterogeneity graphically. Possible differences between the studies were explored by subgroup analyses and meta-regression. The finding was presented using forest plot with respective odds ratio and 95\% confidence intervals. Evidence of publication bias was assessed using both Egger's, and Begg's test with $p$ - value of less than 0.05 as a cutoff point to declare the presence of publication bias $[45,47]$. For the second outcomes, pooled odds ratios with $95 \%$ CI for each factor were used to determine the association between prelacteal feeding practices and its factors (antenatal care, place of delivery, timely initiation of breastfeeding, counselling on infant feeding and place of residences).

\section{Results}

Selection of studies

A total of 492 articles searched through the electronic searches of which 160 duplicated articles were excluded. From the remaining 332 articles, 302 articles were excluded after reading of titles and abstracts. Finally, 30

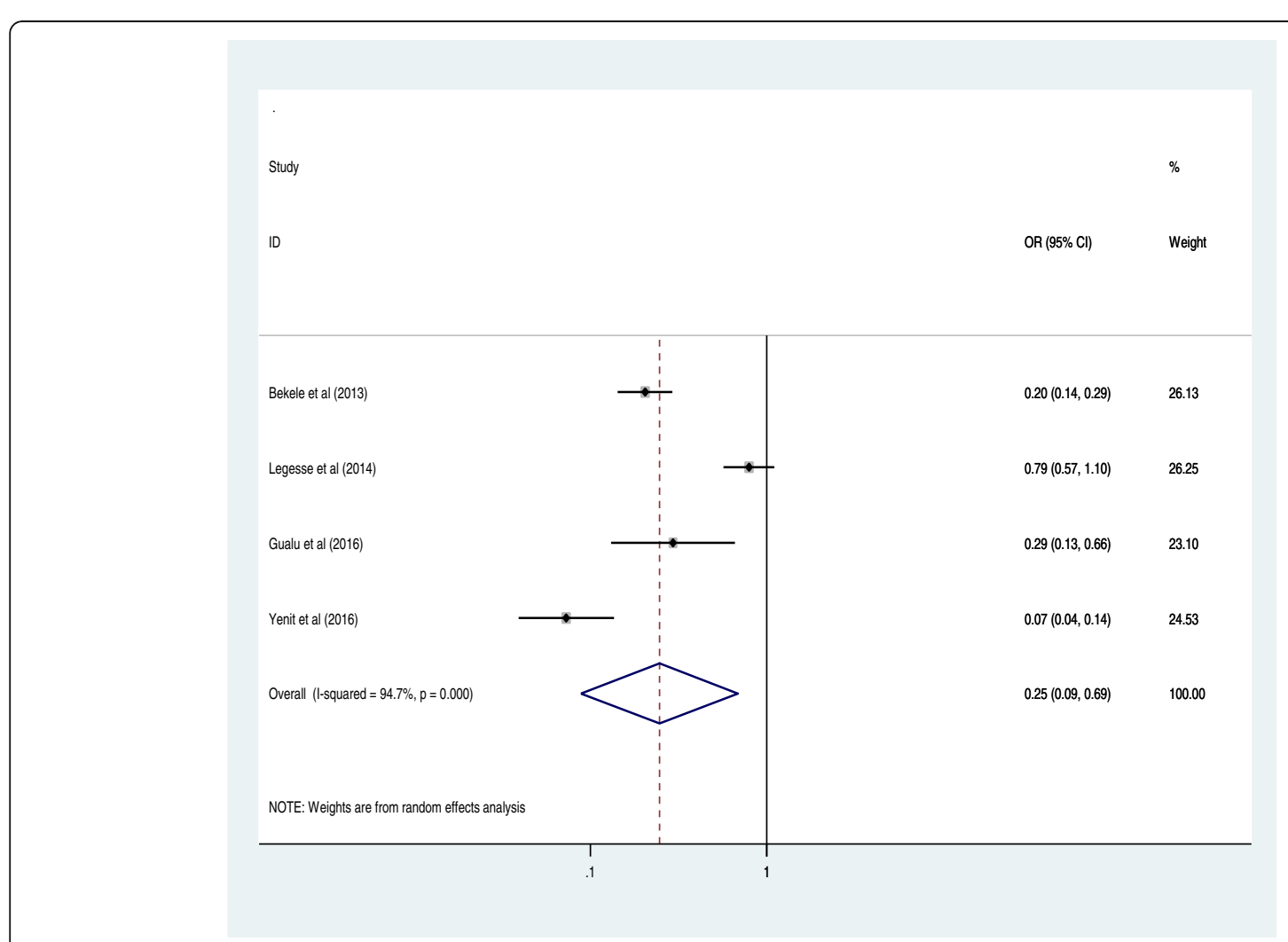

Fig. 3 The pooled odds ratio of the association between antenatal care and prelacteal feeding in Ethiopia 
full text articles were accessed for eligibility criteria. Based on the predefined criteria and after critical appraisal (two articles were excluded [48, 49]), 28 articles were included in the final analysis (Fig. 1).

\section{Characteristics of included studies}

The total of twenty-eight articles was included in this meta-analysis and systematic reviews that met the inclusion criteria. All the included studies were published from 2011 up to 2018. All included studies used cross-sectional study design. A total of 28,435 mothers participated in these studies using an estimated sample size range from 184 [38] up to 6761 [34] to estimate the pooled prevalence prelacteal feeding practice and its associated factors in Ethiopia.

From the total of 28 articles, 15 studies were conducted in Amhara regional state [13, 21, 22, 24, 27-29, 32-34, 36, 37, 41-43]; three studies at South Nations Nationalities and Peoples of Ethiopia national regional state (SNNP) [9, 30, 38]; five studies at Oromia national regional state [7, 20, 35, 39, 40]; two studies at Tigray national regional state $[50,51]$; one study at afar regional state [25]; one study at Binishangul gumuz regional state [31] and one study was conducted at national level in Ethiopia [23]. Twenty-five studies were conducted in the community and the rest three were conducted at the institution based. The results were tabulated according to the prevalence of prelacteal feeding (Table 1).

\section{Pooled prevalence of prelacteal feeding practice in Ethiopia (Meta-analysis)}

The pooled prevalence of prelacteal feeding practice in Ethiopia was $25.29 \%$ (95\% CI 17.43, 33.15) (Fig. 2). As shown in the forest plot below, statistically significant heterogeneity was identified $\left(\mathrm{I}^{2}=99.7 \%\right.$; $\left.p<0.001\right)$ indicating that the use of random effects models for estimating the pooled estimates is applicable. The significant magnitude of the heterogeneity also suggests the need to conduct subgroup analysis to identify the sources of heterogeneity (Fig. 2).

\section{Subgroup analysis}

Subgroup analysis was done based on study area (regions), study years, sampling techniques and study

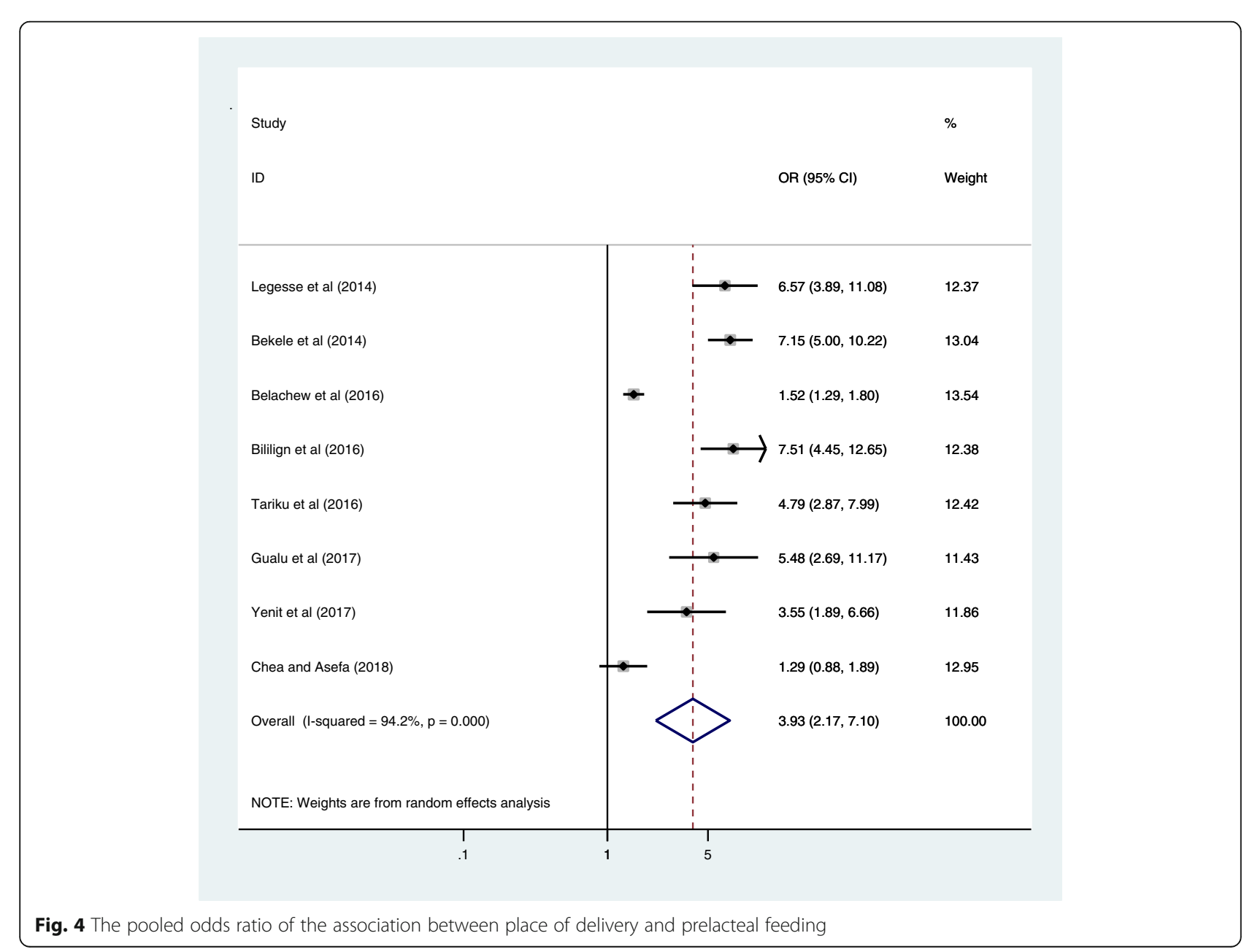




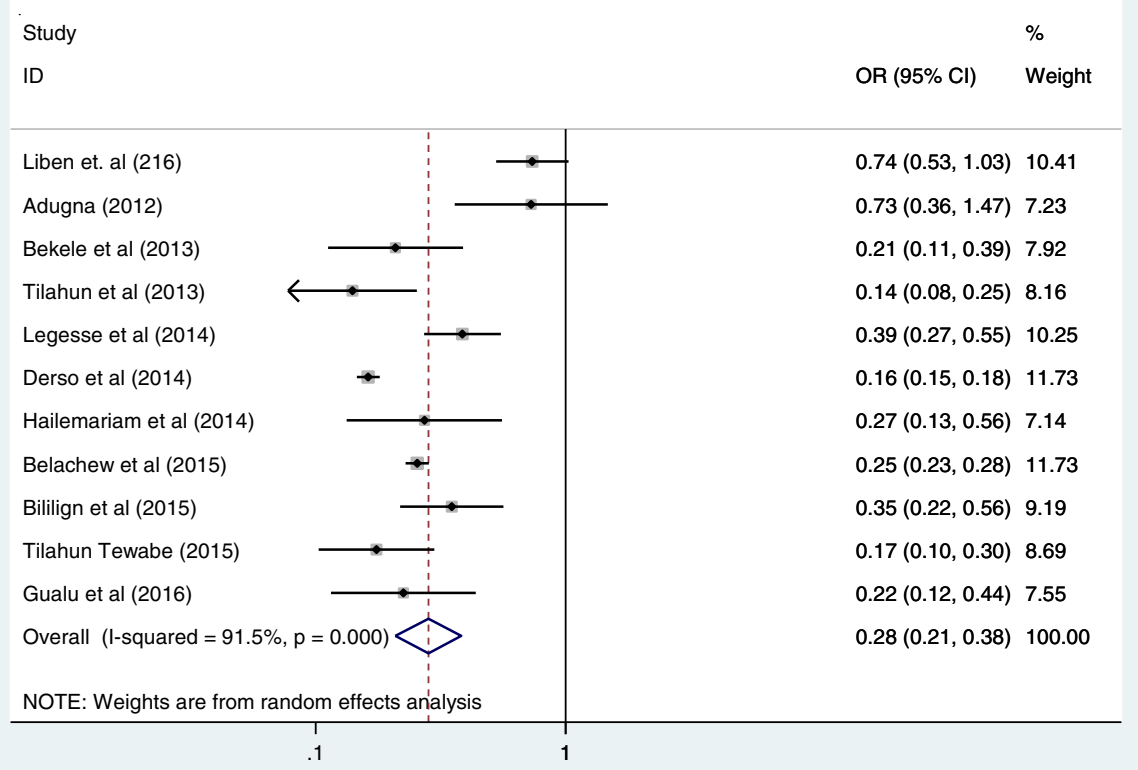

Fig. 5 The pooled odds ratio of the association between counselling on infant feeding and prelacteal feeding

setting to identify the possible source of heterogeneity across studies (Table 2). The subgroup analysis result directed that the source of heterogeneity was not due to the study area, study years, sampling techniques and study setting $(p<0.001)$ (Table 2$)$.

The lowest pooled prevalence of prelacteal feeding practice was indicated in SPNN 17.04\% (95\% CI 5.97, 28.11 ) and the highest was in Oromia region $29.76 \%$
(CI 4.04, 55.49) followed by Amhara region 27.37\% (95\% CI 15.50, 39.25). There was decrement of prelacteal feeding practices starting from 2015 of $18.70 \%$ (95\% CI 14.10, 23.30) (Table 2). In addition to subgroup analysis, publication bias as the source of heterogeneity was also checked using both Begg's and Egger's test. The result of Begg and Egger tests were not identified as the source of heterogeneity pooled

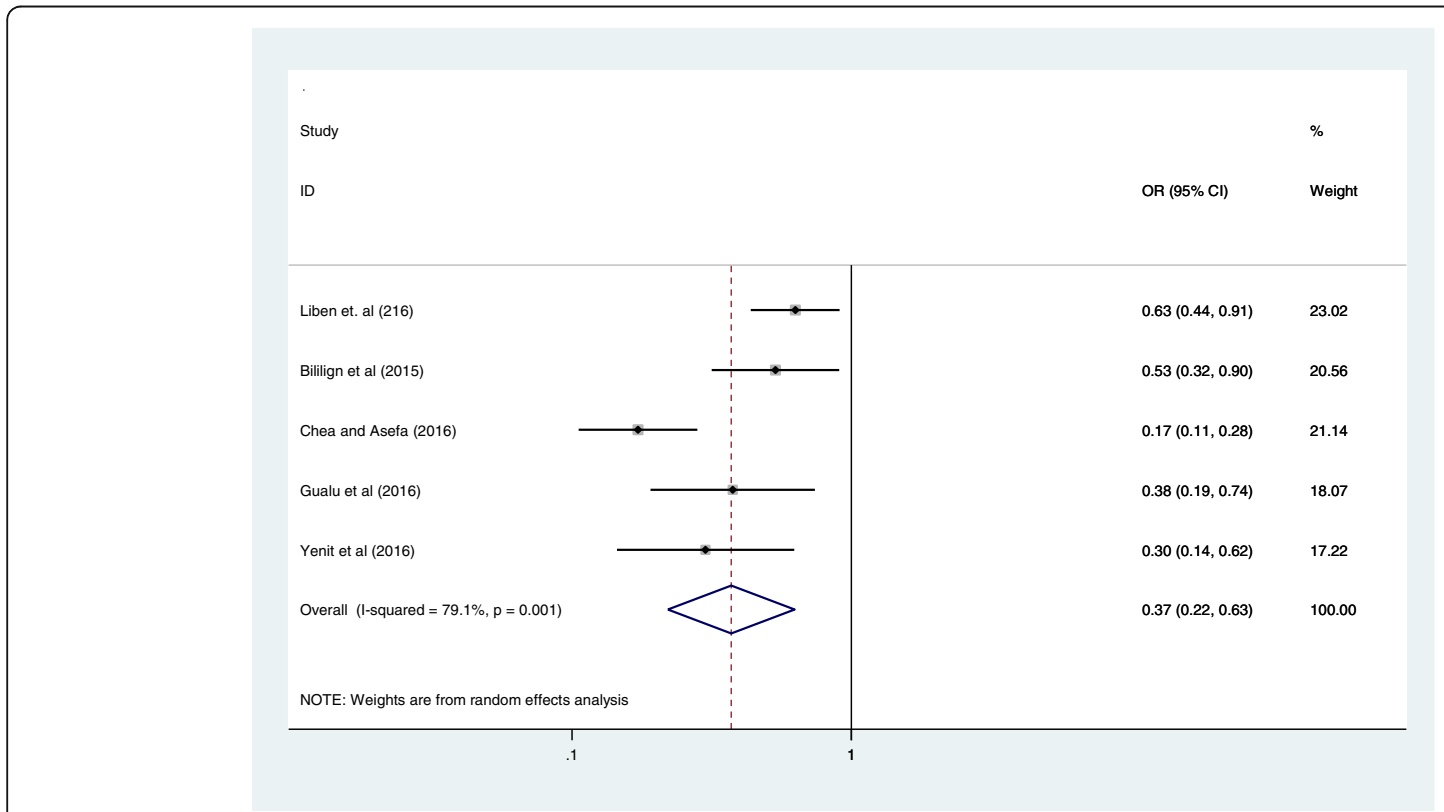

Fig. 6 The pooled odds ratio of the association between time to initiation of breastfeeding and prelacteal feeding 
prevalence of prelacteal feeding practices at $p$ - value of $(p=0.055)$ and $(p=0.181)$ respectively.

\section{Meta regression}

Besides subgroup analysis and publication bias, meta regression was also assumed by considering both continuous and categorical data to identify associated factors of heterogeneity for the pooled prevalence of prelacteal feeding practices. Sample size, study year, study setting and sampling techniques were considered in the meta-regression. However, the meta-regression indicated that the pooled prevalence of prelacteal feeding was not associated with study year, sample size, study setting and sampling techniques (Table 3).

\section{Associated factors of prelacteal feeding practices in Ethiopia}

The overall pooled odds ratio was estimated for different factors reported repeatedly that affect the prelacteal feeding practices in Ethiopia. Antenatal care for index child [7, 22, 24, 28], place of delivery [7, 9, 13, 22-24, 27, 28], counselling about feeding [9, 13, 24, 25, 28], time to initiation of breastfeeding $[7,13,20,22-25,29$, $30,34,41]$ and place of residence $[7,22,23,28]$ were repeatedly reported as significant factors for prelacteal feeding practices.

Antenatal care was significantly associated with prelacteal feeding practices in Ethiopia, odds ratio 0.25 (95\%
CI 0.09, 0.69) (Fig. 3). This indicates that those mothers who had antenatal care for index child $25 \%$ times less likely feed prelacteal feeding than counterparts. The odds of developing prelacteal feeding are 3.93 times higher among mothers who was delivered the child at home compared with institutional delivery $(\mathrm{OR}=3.93$, 95\% CI 2.17, 7.10) (Fig. 4). The odds of prelacteal feeding practices are $37 \%$ times lesser among mothers consoled on prelacteal feeding during pregnancy than did not consoled ( $\mathrm{OR}=0.37$, 95\% CI $0.22,0.63$ ) (Fig. 5). Timely initiation of breastfeeding $28 \%$ times less likely practice prelacteal feeding than those does not initiate breast milk timely within 3 hours (OR $=0.28,95 \% \mathrm{CI}$ $0.21,0.38$ ) (Fig. 6). An urban residence is $47 \%$ times less likely practice prelacteal feeding than rural residence $(\mathrm{OR}=0.47,95 \%$ CI 0.26, 0.86) (Fig. 7).

\section{Discussion}

This systematic review and meta-analysis was conducted to estimate the pooled prevalence of prelacteal feeding practices and its associated factors among mothers having children age less than 5 years in Ethiopia from 2011 up to 2018.

According to this systematic review and meta-analysis, one fourth (25.29\%) of children were given prelacteal foods in Ethiopia. Mothers who had antenatal care for index child, an urban residence and counselling on child feeding practices during pregnancy were less likely to practice

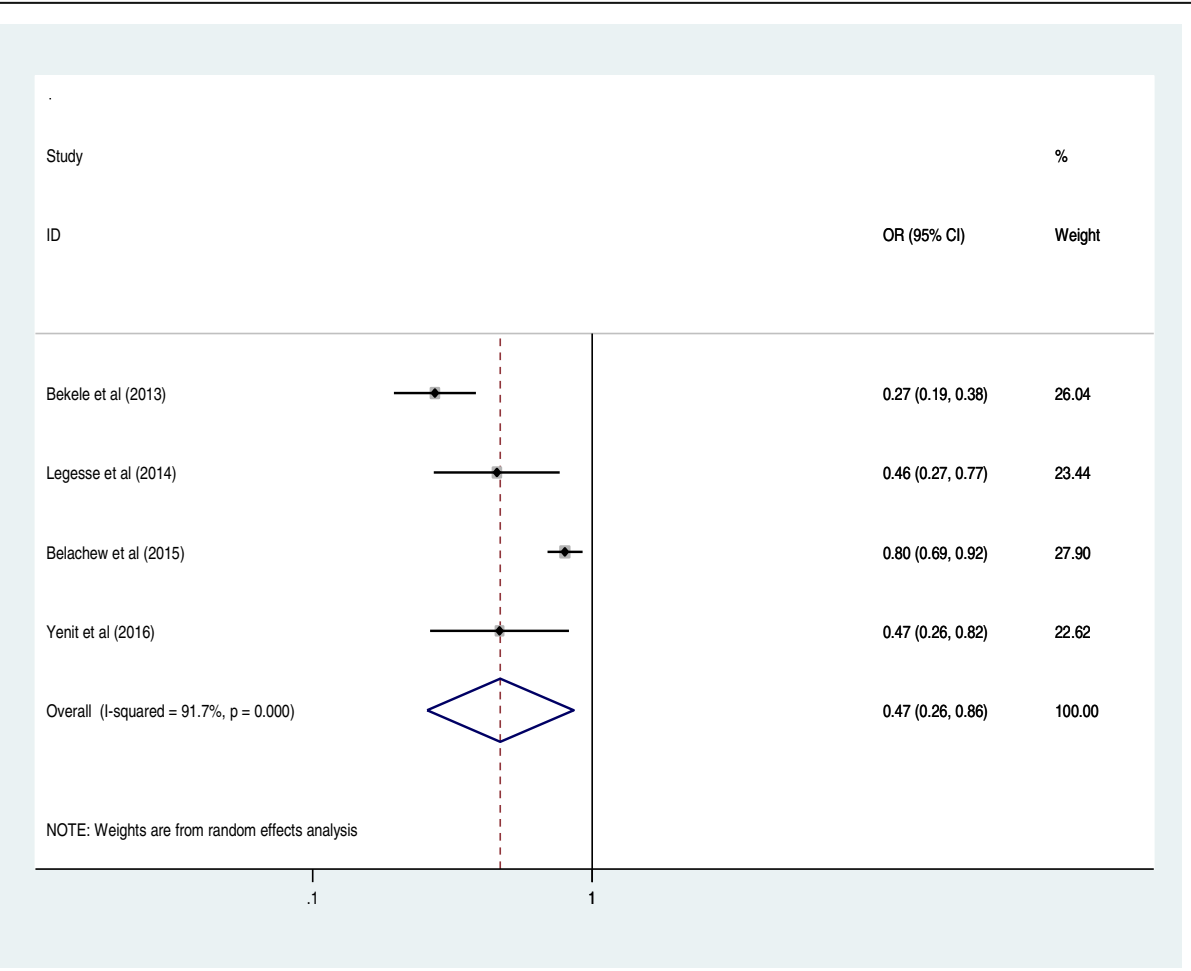

Fig. 7 The pooled odds ratio of the association between place of residence and prelacteal feeding 
prelacteal feeding in Ethiopia. Whereas, mothers who delivered at home gave prelacteal feed than those who delivered in health institutions in Ethiopia.

The prevalence of the prelacteal feeding practice in the current systematic review and meta-analysis is in line with the study conducted in Nepal [3], which it is lower than the study conducted in Nigeria (66.4 and 49.8\%) [52], Sub-Saharan African (32.2\%) [15] and Vietnam (73.3\%) [10]. This disparity may be due to variation of socio-cultural, demographic, and methodological and time of the study across those countries. The magnitude of prelacteal feeding varies across regions in Ethiopia. The highest magnitude was observed in Oromia region followed by Amhara region while the lowest magnitude was observed in southern nation nationalities and peoples of Ethiopia (SNNP). This may be due to cultural difference across regions in Ethiopia. On the other hand, prelacteal feeding practice is decreasing relatively from 2015 and later. This is due to the efforts made by the Ethiopian government and non-government organizations on infant feeding activities during the era of the Millennium Development Goals.

This systematic review and meta-analysis indicated the presence of antenatal care during pregnancy and place of delivery were found to have a statistically significant association with prelacteal feeding practices. Those mothers who had been antenatal care during pregnancy $25 \%$ times less likely to practice prelacteal feeding. This study is in line with the study conducted in Sub-Saharan Africa [15] and in Burkina Faso [53]. The possible reason may be during the period antenatal visit, there is counseling on infant feeding practices since an infant and young child feeding (IYCF) strategy is also the component of the national nutrition strategy in Ethiopia which includes those pregnant women. Women who gave birth at their home is 3.93 times more likely to practice prelacteal feeding than who gave birth at health institutions. This finding is consistent with the study done in Sub-Saharan Africa [15], Nigeria trained study [54], Nigeria population based demographic and health survey [55], in Burkina Faso and South Africa [53]. This may be due to the fact that home delivery is attended by traditional birth attendants who do not have the knowledge of exclusive breastfeeding and the harms of prelacteal feeding practice. Moreover, in Ethiopia, those elders and traditional birth attendants promote prelacteal feeding practices. In health facilities, the early initiation of breastfeeding is practiced, the negative effect of prelacteal feeding and the benefit of exclusive breastfeeding are promoted by health professionals during the periods of postnatal stay. Also mothers who give birth in a health facility are likely to be advised by health professionals about the risks associated with prelacteal feeding practices.

Timely initiation of breastfeeding, counseling about infant feeding practice and living in an urban residence had also the positive impact to decrease prelacteal feeding in Ethiopia. Those mothers' who initiated timely breastfeeding were $28 \%$ less likely to practice prelacteal feeding than their counterparts. There is a close relationship between early initiation and avoiding prelacteal feeds. Mothers who received counseling on infant feeding during the perinatal period were less likely to offer prelacteal food than who did not receive counseling. Similar findings was reported in India [56]. This might be due to that counseling is the tool to change the behaviors of mothers to wards prelacteal feeding practice during the time of pregnancy.

Living in an rural residence is 53\% times more likely feed prelacteal foods than urban residences in Ethiopia. This is in line with the study done in Nigeria $[54,55]$. This might be due to rural residences are relatively having low awareness about the risk of prelacteal feeding and also cultural practices are more common in rural communities.

These studies have certain limitations, which includes articles published in English language, all are cross-sectional articles, and some of the regions did not include because of lack of research.

\section{Conclusion}

In Ethiopia, one in four mothers gave prelacteal foods for their children. Mothers who gave birth at home are more prone to give prelacteal foods. Whereas, antenatal care, timely initiation of breastfeeding, counseling on infant feeding and living in an urban residence decreased prelacteal feeding practices in Ethiopia. On the contrary, home delivery practice increased the risk of prelacteal feeding in Ethiopia. Therefore, the government and health institutions should focus on awareness creation about risk of prelacteal feeding, increase antenatal care service, promote institutional delivery, recommend timely initiation of breastfeeding and increase the counseling service about infant feeding during pregnancies.

\section{Abbreviations \\ ANC: Antenatal care; Cl: Confidence interval; EBF: Exclusive breastfeeding; IYCF: Infant and young child feeding; OR: Odds ratio; PLF: Prelacteal feeding practice; SNNP: South Nations Nationalities and Peoples; WHO: World Health Organization}

\section{Acknowledgments \\ We would like to thank all authors of studies included in this systematic review and meta-analysis.}

\section{Author contributions}

HT and AN involved in the design, selection of study, data extraction, statistical analysis and developing the initial drafts of the manuscript. HT, TG, WW, and MY involved in quality assessment. HT, AN and WW prepared and 
revising subsequent drafts. HT and AN prepared the final draft of the manuscript. All authors read and approved the final draft of the manuscript.

\section{Funding}

Not applicable

\section{Availability of data and materials}

The data sets analyzed during the current study are available from the corresponding author upon reasonable request.

\section{Ethics approval and consent to participate}

Not applicable

\section{Consent for publication}

Not applicable

\section{Competing interests}

The authors declare that they have no competing interests.

\section{Publisher's Note}

Springer Nature remains neutral with regard to jurisdictional claims in published maps and institutional affiliations.

\section{Author details Markos University, Debre Markos, Ethiopia. \\ Received: 26 July 2018 Accepted: 19 November 2018 Published online: 28 November 2018}

${ }^{1}$ Department of Human Nutrition and Food Sciences, College of Health Science, Debre Markos University, Debre Markos, Ethiopia. ${ }^{2}$ Department of Midwifery, College of Health Science, Debre Markos University, Debre Markos, Ethiopia. ${ }^{3}$ Department of Public Health, College of Health Science, Debre

\section{References}

1. Jimoh AO, Adaji SE, Adelaiye HA, Olorukooba AA, Garba C, Mfuh AL, et al. Factors associated with prelacteal feeding practices in a rural northern Nigerian setting. S Afr J Clin Nutr. 2018:31:37-42.

2. WHO. Infant and young child feeding: model chapter for textbooks for medical students and allied health professionals. In: Infant and Young Child Feeding: Model Chapter for Textbooks for Medical Students and Allied Health Professionals; 2009.

3. Khanal V, Adhikari M, Sauer K, Zhao Y. Factors associated with the introduction of prelacteal feeds in Nepal: findings from the Nepal demographic and health survey 2011. Int Breastfeed J. 2013:8:9.

4. Jones AD, Ickes SB, Smith LE, Mduduzi MN, Chasekwa B, Heidkamp RA, et al. World health organization infant and young child feeding indicators and their associations with child growth: a synthesis of recent findings. Matern Child Nutr. 2014;10:1-17.

5. Victora CG, Bahl R, Barros AJ, França GV, Horton S, Krasevec J, Murch S, et al. Breastfeeding in the 21st century: epidemiology, mechanisms, and lifelong effect. Lancet. 2016:387:475-90.

6. Yonas F, Asnakew M, Wondafrash M, Abdulahi M. Infant and young child feeding practice status and associated factors among mothers of under 24-month-old children in Shashemene Woreda, Oromia region, Ethiopia. Open Access Libr J. 2015;2:10.

7. Bekele Y, Mengistie B, Mesfine F. Prelacteal feeding practice and associated factors among mothers attending immunization clinic in Harari region public health facilities, Eastern Ethiopia. Open J Prev Med. 2014:4:529-34.

8. World Health Organization: Global health risks: mortality and burden of disease attributable to selected major risks. Geneva, Switzerland: the World Health Organization; 2009.

9. Chea N, Asefa A. Prelacteal feeding and associated factors among newborns in rural Sidama, South Ethiopia: a community based cross-sectional survey. Int Breastfeed J. 2018;13:7.

10. Nguyen PH, Keithly SC, Nguyen NT, Nguyen TT, Tran LM, Hajeebhoy N. Prelacteal feeding practices in Vietnam: challenges and associated factors. BMC Public Health. 2013;13:932

11. Leach A, McArdle T, Banya W, Krubally O, Greenwood A, Rands C, et al. Neonatal mortality in a rural area of the Gambia. Ann Trop Paediatr. 1999;19:33-43.
12. (EHNRI) EHaNRI: Nutritional Baseline Survey Report for the National Nutrition Program of Ethiopia. 2010.

13. Bililign N, Kumsa H, Mulugeta M, Sisay Y. Factors associated with prelacteal feeding in north eastern Ethiopia: a community based cross-sectional study. Int Breastfeed J. 2016;11:13.

14. Roy MP, Mohan U, Singh SK, Singh VK, Srivastava AK. Determinants of prelacteal feeding in rural northern India. Int J Prev Med. 2014;5:658.

15. Berde AS, Ozcebe H. Risk factors for prelacteal feeding in sub-Saharan Africa: a multilevel analysis of population data from twenty-two countries. Public Health Nutr. 2017;20:1953-62.

16. Hossain MM, Radwan MM, Arafa SA, Habib M, DuPont HL. Prelacteal infant feeding practices in rural Egypt. J Trop Pediatr. 1992;38:317-22.

17. Ogah A, Ajayi A, Akib S, Okolo S. A cross-sectional study of pre-lacteal feeding practice among women attending Kampala International University teaching hospital maternal and child health clinic, Bushenyi, Western Uganda. Asian J Med Sci. 2012;4:79-85.

18. CSA ICF International. Ethiopia demographic and health survey 2011. In: Addis Ababa, Ethiopia and Calverton, Maryland. USA: Central Statistical Agency and ICF International; 2012. p. 430.

19. Alemu ZA, Ahmed AA, Yalew AW, Birhanu BS, Zaitchik BF. Individual and community level factors with a significant role in determining child height-for-age Z score in east Gojjam zone, Amhara regional state, Ethiopia: a multilevel analysis. Arch Public Health. 2017;75:27.

20. Hailemariam TW, Adeba E, Sufa A. Predictors of early breastfeeding initiation among mothers of children under 24 months of age in rural part of West Ethiopia. BMC Public Health. 2015;15:1076.

21. Mekuria G, Edris M. Exclusive breastfeeding and associated factors among mothers in Debre Markos, Northwest Ethiopia: a cross-sectional study. Int Breastfeed J. 2015;10:1

22. Legesse M, Demena M, Mesfin F, Haile D. Prelacteal feeding practices and associated factors among mothers of children aged less than 24 months in Raya kobo district, north eastern Ethiopia: a cross-sectional study. Int Breastfeed J. 2014;9:189.

23. Belachew $A B$, Kahsay $A B$, Abebe $Y G$. Individual and community-level factors associated with introduction of prelacteal feeding in Ethiopia. Arch Public Health. 2016:74:6.

24. Gualu T, Dilie A, Haile D, Abate A. Determinants of prelacteal feeding practice among postpartum mothers in Debre Markos town, Amhara regional state, Ethiopia, 2016. Nutr Diet Suppl. 2017;9:97-102.

25. Liben ML. Determinants of early initiation of breastfeeding among mothers: the case of Raya Kobo District, Northeast Ethiopia: a cross-sectional study. Int J Nutr Food Sci. 2015:4:289-94.

26. Liben ML, Wuneh AG, Zepro NB, Mulugeta A. Factors associated with prelacteal feeding in afar regional state, northeastern Ethiopia: a cross sectional study. Int J Res. 2017;5:116-27.

27. Tariku A, Biks GA, Wassie MM, Gebeyehu A, Getie AA. Factors associated with prelacteal feeding in the rural population of Northwest Ethiopia: a community cross-sectional study. Int Breastfeed J. 2016;11:14.

28. Yenit MK, Genetu H, Tariku A. Infant feeding counseling and knowledge are the key determinants of prelacteal feeding among HIV exposed infants attending public hospitals in Ethiopia. Arch Public Health. 2017:75:23.

29. Tewabe T. Timely initiation of breastfeeding and associated factors among mothers in Motta town, east Gojjam zone, Amhara regional state, Ethiopia 2015: a cross-sectional study. BMC Pregnancy Childbirth. 2016;16:314.

30. Adugna DT. Women's perception and risk factors for delayed initiation of breastfeeding in Arba Minch Zuria, Southern Ethiopia. Int Breastfeed J. 2014;9:8

31. Ayana D, Tariku A, Feleke A, Woldie H. Complementary feeding practices among children in Benishangul Gumuz region, Ethiopia. BMC Res Notes. 2017;10:335.

32. Bimerew A, Teshome M, Kassa GM. Prevalence of timely breastfeeding initiation and associated factors in Dembecha district, north West Ethiopia: a cross-sectional study. Int Breastfeed J. 2016;11:28.

33. Demilew $Y M$, Tafere TE, Abitew DB. Infant and young child feeding practice among mothers with 0-24 months old children in slum areas of Bahir Dar City, Ethiopia. Int Breastfeed J. 2017;12:26.

34. Derso T, Biks GA, Tariku A, Tebeje NB, Gizaw Z, Muchie KF, Shimeka A, Kebede $Y$, Abebe SM, Yitayal M. Correlates of early neonatal feeding practice in Dabat HDSS site, northwest Ethiopia. Int Breastfeed J. 2017:12:25.

35. Egata G, Berhane $Y$, Worku A. Predictors of non-exclusive breastfeeding at 6 months among rural mothers in East Ethiopia: a community-based analytical cross-sectional study. Int Breastfeed J. 2013:8:8 
36. Fentahun W, Wubshet M, Tariku A. Undernutrition and associated factors among children aged 6-59 months in east Belesa District, Northwest Ethiopia: a community based cross-sectional study. BMC Public Health. 2016;16:506.

37. Genetu H, Yenit MK, Tariku A. Breastfeeding counseling and support are associated with continuous exclusive breastfeeding from one week to six months of age among HIV exposed infants in North Gondar zone, Ethiopia: a cross-sectional study. Int Breastfeed J. 2016;12:21.

38. Haile $\mathrm{D}$, Setegn T, Biadgilign $\mathrm{S}$. Adherence to WHO breastfeeding guidelines among HIV positive mothers in southern Ethiopia: implication for intervention. Pediatr Health Med Ther. 2015;6:87.

39. Asfaw M, Wondaferash M, Taha M, Dube L. Prevalence of undernutrition and associated factors among children aged between six to fifty nine months in Bule Hora district, South Ethiopia. BMC Public Health. 2015;15:41.

40. Zenebu BB, Belayneh KG, Alayou G, Ahimed A, Bereket C, Abreham A, et al. Knowledge and practice of mothers towards exclusive breastfeeding and its associated factors in ambo Woreda west Shoa zone Oromia region, Ethiopia. Epidemiol: Open Access. 2015;5:182.

41. Tilahun G, Degu G, Azale T, Tigabu A. Prevalence and associated factors of timely initiation of breastfeeding among mothers at Debre Berhan town, Ethiopia: a cross-sectional study. Int Breastfeed J. 2016;11:27.

42. Abebe Z, Zelalem Anlay D, Biadgo B, Kebede A, Melku T, Enawgaw B, et al. High prevalence of undernutrition among children in Gondar town, Northwest Ethiopia: A Community-Based Cross-Sectional Study. Int J Pediatr. 2017;2017:9.

43. Asemahagn MA. Determinants of exclusive breastfeeding practices among mothers in azezo district, northwest Ethiopia. Int Breastfeed J. 2016;1 1:22.

44. Moher D, Liberati A, Tetzlaff J, Altman DG. Preferred reporting items for systematic reviews and meta-analyses: the PRISMA statement. Ann Intern Med. 2009;151:264-9.

45. Begg CB, Mazumdar M. Operating characteristics of a rank correlation test for publication bias. Biometrics. 1994;50:1088-1101.

46. Rücker G, Schwarzer G, Carpenter JR, Schumacher M. Undue reliance on I 2 in assessing heterogeneity may mislead. BMC Med Res Methodol. 2008;8:79.

47. Egger M, Smith GD, Schneider M, Minder C. Bias in meta-analysis detected by a simple, graphical test. Bmj. 1997;315:629-34.

48. Girma T. Traditional newborn care in Jimma town, Southwest Ethiopia. Ethiop J Health Sci. 2008;18:79-86.

49. Rogers NL, Abdi J, Moore D, Nd'iangui S, Smith L, Carlson AJ, et al. Colostrum avoidance, prelacteal feeding and late breast-feeding initiation in rural northern Ethiopia. Public Health Nutr. 2011;14:2029-36.

50. Teka B, Assefa H, Haileslassie K. Prevalence and determinant factors of exclusive breastfeeding practices among mothers in Enderta woreda, Tigray, North Ethiopia: a cross-sectional study. Int Breastfeed J. 2015;10:2.

51. Alemayehu M, Abreha K, Yebyo H, Zemichael K, Gebremichael H. Factors associated with timely initiation and exclusive breastfeeding among mothers of Axum town, northern Ethiopia. Sci J Public Health. 2014;2:394-401.

52. Berde AS, Yalcin SS. Determinants of early initiation of breastfeeding in Nigeria: a population-based study using the 2013 demograhic and health survey data. BMC Pregnancy Childbirth. 2016;16:32.

53. Engebretsen IMS, Nankabirwa V, Doherty T, Diallo AH, Nankunda J, Fadnes $L T$, et al. Early infant feeding practices in three African countries: the PROMISE-EBF trial promoting exclusive breastfeeding by peer counsellors. Int Breastfeed J. 2014;9:19.

54. Agho KE, Ogeleka P, Ogbo FA, Ezeh OK, Eastwood J, Page A. Trends and predictors of prelacteal feeding practices in Nigeria (2003-2013). Nutrients. 2016;8:462.

55. Berde AS, Yalcin SS, Ozcebe H, Uner S, Caman OK. Determinants of prelacteal feeding practices in urban and rural Nigeria; a population-based cross-sectional study using the 2013 Nigeria demographic and health survey data. Afr Health Sci. 2017;17:690-9.

56. Patel A, Banerjee A, Kaletwad A. Factors associated with prelacteal feeding and timely initiation of breastfeeding in hospital-delivered infants in India. J Hum Lact. 2013;29:572-8.

\section{Ready to submit your research? Choose BMC and benefit from:}

- fast, convenient online submission

- thorough peer review by experienced researchers in your field

- rapid publication on acceptance

- support for research data, including large and complex data types

- gold Open Access which fosters wider collaboration and increased citations

- maximum visibility for your research: over $100 \mathrm{M}$ website views per year

At BMC, research is always in progress.

Learn more biomedcentral.com/submissions 